\title{
Redox-Polymers Enable Uninterrupted Day/Night Photo-Driven Electricity Generation in Biophotovoltaic Devices
}

\author{
Libertus Darus, ${ }^{\mathrm{a}, \mathrm{b}}$ Takuya Sadakane, ${ }^{\mathrm{c}}$ Pablo Ledezma, ${ }^{\mathrm{a}}$ Seiya Tsujimura, ${ }^{\mathrm{c}}$ Isioma Osadebe, ${ }^{\mathrm{d}}$ \\ Dónal Leech, ${ }^{\mathrm{d}, *}$ Lo Gorton, ${ }^{\mathrm{e}}$ and Stefano Freguia ${ }^{\mathrm{a}, \mathrm{z}}$ \\ ${ }^{a}$ Advanced Water Management Centre, The University of Queensland, St. Lucia QLD 4072, Australia \\ ${ }^{b}$ Department of Agricultural Technology, State Polytechnic of Pontianak, Jl. Ahmad Yani, \\ Pontianak-West Borneo 78124, Indonesia \\ ${ }^{c}$ Division of Materials Science, Faculty of Pure and Applied Sciences, University of Tsukuba, Tsukuba, \\ Ibaraki 305-8573, Japan \\ ${ }^{d}$ School of Chemistry, National University of Ireland Galway, Galway, Ireland \\ ${ }^{e}$ Department of Analytical Chemistry/Biochemistry and Structural Biology, Lund University, SE-22100 Lund, Sweden
}

\begin{abstract}
Biophotovoltaic devices modified with immobilized polymeric osmium/azine redox-mediators exhibited a considerable electrical output enhancement (64/43-fold under light/dark conditions, respectively). More importantly, the systems exhibited uninterrupted current generation at same magnitude levels during day/night cycles, paving the way toward solar energy conversion bio-panels that will not require energy storage peripherals.

(c) The Author(s) 2016. Published by ECS. This is an open access article distributed under the terms of the Creative Commons Attribution Non-Commercial No Derivatives 4.0 License (CC BY-NC-ND, http://creativecommons.org/licenses/by-nc-nd/4.0/), which permits non-commercial reuse, distribution, and reproduction in any medium, provided the original work is not changed in any way and is properly cited. For permission for commercial reuse, please email: oa@electrochem.org. [DOI: 10.1149/2.0091703jes] All rights reserved.

(cc) BY-NC-ND
\end{abstract}

Manuscript submitted September 14, 2016; revised manuscript received November 22, 2016. Published December 6, 2016. This paper is part of the JES Focus Issue on Biological Fuel Cells.

Biophotovoltaic systems (BPVs), also known as biosolar or photobioelectrochemical cells, are an emerging biotechnology for the light-driven renewable production of electricity. BPVs rely fundamentally on the ability of various photosynthetic microorganisms, primarily cyanobacteria, to divert electrons from certain metabolic pathways - the exact routes remain a matter of debate ${ }^{1}$ - onto insoluble electron acceptors i.e. solid electrodes. To date however, low electrical outputs and polarity reversal during illumination remain strong limitations toward any real-world practical implementation of BPVs. ${ }^{2-4}$ Previous research efforts have focused on maximising current output by utilising soluble electron mediators which allow for electron transfer from the enzymatic active sites within the cells to the electrodes ${ }^{5-9}$ Nevertheless, the use of soluble mediators requires continuous replenishment, which makes their use unsustainable, costly and in some cases even harmful to the environment. ${ }^{7,10}$ Recent investigations in the field of bioelectronics have demonstrated that electron shuttling via immobilized redox mediators such as polymeric osmium complexes ${ }^{11,12}$ and polymeric azines ${ }^{13-15}$ (e.g. polymethylene blue (PMB), polythionine or polymethylene green (PMG)) can be a stable and efficient alternative. ${ }^{16}$ Indeed, by immobilising polymeric osmium complexes onto working electrodes, the biocatalytic performance of pure cultures of the cyanobacterium Leptolyngbya ${ }^{17}$ and the microalga Paulschulzia ${ }^{18}$ have been significantly improved.

For the practical implementation of BPVs however, the use of a microbial consortium is considered advantageous over axenic cultures, because consortia typically produce higher electrical outputs and have higher resilience to variable physicochemical and process conditions. ${ }^{1}$ In this study, we report for the first time on the significant enhancement of current output by a mat-building marine photosynthetic consortium dominated by cyanobacteria, through immobilization of polymerized osmium complexes or azine mediators onto photoelectrochemical cell anodes. The electrode modifications resulted not only in significant output enhancement, but also in the disappearance of current reversals, leading to uninterrupted day/night anodic current production at similar magnitude levels.

\section{Experimental}

Setup preparations.-The experiments were conducted in 3electrode photoelectrochemical cells of $70 \mathrm{~mL}$ volume with plain or modified graphite rods (diameter $4.73 \mathrm{~mm}$ ) as working electrodes. For each cell, the side of the graphite rod was covered with a rubber sheath, leaving only the base as working surface (area: $17.6 \mathrm{~mm}^{2}$ ), whilst a Ti wire (length $4 \mathrm{~cm}$, diameter $0.5 \mathrm{~mm}$ ) and a $\mathrm{KCl}$-saturated $\mathrm{Ag} / \mathrm{AgCl}$ electrode $(+0.197 \mathrm{~V}$ vs SHE$)$ were used as counter and reference electrodes, respectively. An electrogenic photosynthetic marine biofilm dominated by cyanobacteria $(52 \%)$ with the rest consisting mainly of heterotrophs (48\%) was scraped off a working photoelectrochemical cell (see enrichment process and full community composition in Darus et al. ${ }^{19}$ ) and used as inoculum. The electrochemical cells were operated inside a dark box in batch mode, fed with filtersterilized modified $\mathrm{F} 2$ medium $^{4}$ at $\mathrm{pH} 7.8 \pm 2$ containing $40 \mathrm{mM}$ $\mathrm{NaHCO}_{3}$ (as sole carbon source and $\mathrm{pH}$ buffer) and illuminated by white LED lights at $29 \mathrm{~W} \mathrm{~m}^{-2}$ in circadian cycles of $4 \mathrm{~h}$ day/4 h night. The experiments were maintained at $24 \pm 1{ }^{\circ} \mathrm{C}$ inside the box using a fan.

Electrode modifications.-Prior to modification, the bases of the graphite rods were polished with $1 \mu \mathrm{m}$ alumina powder for 3 min, rinsed and sonicated with distilled water. The polymeric osmium complexes [Os(2,2'-bipyridine $)_{2}$ (polyvinyl-imidazole $\left.)_{10} \mathrm{Cl}\right] \mathrm{Cl}$ (hereafter referred to as Os-1) and [Os(4,4'-dimethyl-2,2'bipyridine $)_{2}$ (polyvinyl-imidazole $\left.)_{10} \mathrm{Cl}\right] \mathrm{Cl}$ (hereafter Os-2) were prepared according to previous reports by Ohara et al. ${ }^{20}$ and Osadebe and Leech, ${ }^{11}$ respectively. Individually, $25 \mu \mathrm{L}$ solution of $7 \mathrm{mg} \mathrm{mL}^{-1}$ polymeric osmium complex (in distilled water) was mixed with $5 \mu \mathrm{L}$ solution of $7 \mathrm{mg} / \mathrm{mL}$ poly (ethylene glycol) diglycidyl ether (PEGDGE) (Sigma-Aldrich, Australia). $5 \mu \mathrm{L}$ of the mixture was applied evenly on the base of the graphite rod, dried for $12 \mathrm{~h}$ at room temperature and cured for another $24 \mathrm{~h}$ at $8^{\circ} \mathrm{C}$.

For the azine redox-mediators, $1 \mathrm{mM}$ monomer solutions of methylene green (MG), methylene blue (MB) (both from Tokyo Chemical Industry Corp, Japan) and thionine (Sigma-Aldrich, Australia) were prepared individually by dissolving in $100 \mathrm{mM}$ PBS buffer at $\mathrm{pH} 7.0$ and $100 \mathrm{mM} \mathrm{NaNO}_{3}$. The monomer electro-polymerization was then carried out by immersion of the graphite rods' working surfaces in 


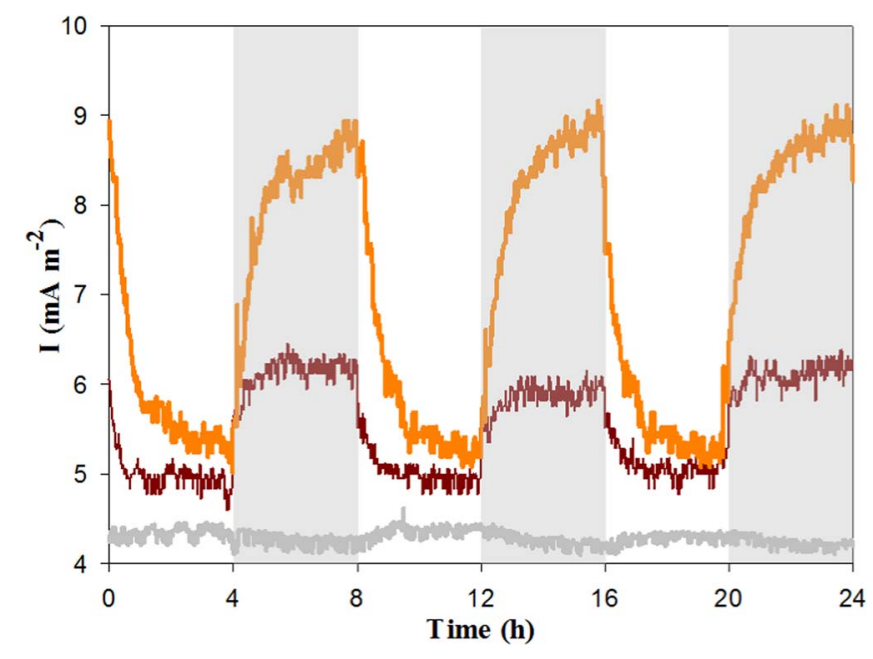

Figure 1. Chronoamperometric anodic current profile of unmodified electrochemical cells: abiotic (gray), first biotic reactor (orange) and duplicate biotic reactor (maroon).

the monomer solution and undertaking cyclic voltammetry $(\mathrm{CV})$ from -0.2 to $+1.4 \mathrm{~V}$ vs SHE for 10 cycles at $10 \mathrm{mV} \mathrm{s}^{-1}$, followed by immersion in distilled water for $\geq 3 \mathrm{~h}$ to remove any residual monomer.

Evaluations.-The surface modification was confirmed by cyclic voltammetry $(\mathrm{CV})$, conducted between 0 and $+0.8 \mathrm{~V}$ vs SHE at a scan rate of $10 \mathrm{mV} \mathrm{s}^{-1}$, with a multichannel potentiostat (CHI1000B, $\mathrm{CH}$ Instruments, USA). Moreover, the electrochemical performance of biotic modified anodes, and abiotic and biotic unmodified controls was evaluated by chronoamperometry (CA) until steady-state was observed (approx. 5 days or 15 light/dark cycles) and for a further 9 cycles (for the duplicate reactors), which is the data hereby presented. Additionally, CA profiles of abiotic PMB-modified anode were conducted. CA experiments were run on all electrochemical cells at a poised anode potential of $+0.6 \mathrm{~V}$ vs SHE to maximize electron extraction from photosynthetic and respiratory electron transport chains. All experiments were run in duplicate independent electrochemical cells.

\section{Results and Discussion}

To confirm the role of the consortium on current generation, the responses of abiotic and biotic unmodified anodes were first investigated via CA. As shown in Fig. 1, no current oscillation was observed for the abiotic unmodified anode. Following inoculation, two cells with unmodified electrodes produced a small amount of current, which was higher at night ( 9 and $6 \mathrm{~mA} \mathrm{~m}^{-2}$ respectively) than during the day ( $5 \mathrm{~mA} \mathrm{~m}^{-2}$ for both). This day/night pattern has previously been observed with other photosynthetic microbial consortia ${ }^{2,21}$ and explained by the detrimental effect of photosynthetically-evolved oxygen during illumination and the higher rates of anodic respiration (of organics produced from fixed $\mathrm{CO}_{2}$ during the day) in the dark. $^{21}$

To enhance the extracellular electron transfer (EET) of the microbial consortium, various redox polymers were immobilized onto the anode surfaces, with CVs subsequently conducted to confirm that the modifications were successful. Redox peaks were observed for both polymeric osmium complexes (Fig. S2A), whereas increased anodic and cathodic currents across the scan range were observed with polymeric azine mediators (Fig. S2B), confirming the successful immobilization of the electroactive material onto the working electrode surfaces.

The sustained day/night anodic current in all modified anodes was furthermore confirmed by $\mathrm{CV}$ conducted in day and night conditions (after reaching steady-state). As shown in Fig. 2, at a potential
$+0.6 \mathrm{~V}$ vs SHE, the biotic day/night anodic current output was higher than the abiotic one for tested modifications. Moreover, at this potential the day-time anodic current was higher than at night. These clear (albeit not massive) differences in biocatalytic current confirm the biological nature of current generation in these biophotovoltaic systems (as shown in Fig. 3). In contrast, the chronoamperometric profile of an abiotic PMB-modified anode (shown in Fig. S4) exhibited reductive current outputs after reaching steady-state conditions, further confirming that the oxidative currents observed on the biotic PMB-modified anode (as shown in Fig. S2B and Fig. 3) were due to biocatalytic activity.

With the help of these modifications, and as shown in Fig. 3 and Fig. S1 for the duplicate reactors (see Supporting Information), the anodic current output increased during both day and night. Under illumination, the enhancement was $64 \pm 6,19 \pm 5,21 \pm 2,22$ \pm 0 and $20 \pm 2$ times, respectively for Os-1, Os-2, polythionine, PMB and PMG, while during the night the improvement was lower but still $43 \pm 8,13 \pm 6,14 \pm 5,15 \pm 4$ and $13 \pm 3$ times correspondingly. Interestingly, the use of these polymeric mediators in both repetitions led to slightly higher anodic currents during the day than at night (see Fig. 3 and S1), which is a reversal of the behavior previously observed without mediators in Fig. 1. This phenomenon suggests that photosynthetic microorganisms are able to use extracellular anodes as terminal electron acceptors for the photosynthetic metabolism. ${ }^{19}$

The better performance of the redox-polymer-bearing anodes compared to the controls may be attributed to the formation of threedimensional multi-layers of electroactive material, maximising the electrical wiring between the biocatalysts and their terminal electron acceptor. ${ }^{14,22,23}$ With regards to the polymeric osmium complexes, a considerably higher current generation was observed with Os-1, reaching $320 \pm 28 \mathrm{~mA} \mathrm{~m}^{-2}$ under illumination and $317 \pm 29$ $\mathrm{mA} \mathrm{m}^{-2}$ in the dark, whilst Os-2 systems reached only $93 \pm 23 \mathrm{~mA}$ $\mathrm{m}^{-2}$ and $89 \pm 20 \mathrm{~mA} \mathrm{~m}^{-2}$ for day/night conditions respectively. These significant differences may be explained by the higher redox potential of Os-1 (+0.428 V vs. SHE) versus Os-2 (+0.188 V vs SHE; see Fig. 2 ), which may $(i)$ speed up electron harvesting from the photosynthetic electron transport chain ${ }^{18}$ and/or ( $\left.i i\right)$ lead to a lower likelihood for Os-1 to get re-oxidized by photosynthetically-evolved oxygen, a problem that severely inhibits EET, as previously demonstrated. ${ }^{21}$ Similar effects were previously reported for pure cyanobacterial ${ }^{17}$ and algal species. ${ }^{18}$ Moreover, although the deposition methods and amounts of Os-mediator utilized were identical, it is also possible that (iii) different concentrations of Os-1 and Os-2 were ultimately immobilized onto the studied electrode surfaces (as hinted by the CVs in Fig. S2A). Therefore, the performance differences could be attributed to the combination of all three factors, prompting a need for future research.

Nevertheless, the biological cultures - which had been electrochemically active for years without polymers in other reactors ${ }^{4,19}$ responded immediately and stably (see Fig. S3 for longer-term performance) to the redox-polymer additions with consistent differences in electrical output throughout the length of the experiments, indicating lack of any toxicity effects and demonstrating the stability of the modifications over time (as previously demonstrated for $>6$ months in physiological solutions ${ }^{24}$ ).

\section{Conclusions}

Electrochemical testing indicates the chosen polymeric mediators were successfully immobilized onto anode surfaces and significantly enhanced light-driven electron transfer from microorganisms to electrodes. The co-immobilization of these polymeric mediators and photosynthetic consortia as biocatalysts led to significant current enhancement and uninterrupted anodic current generation during day/night cycles, an important step toward the implementation of electricity production by biophotovoltaic systems in niche contexts. 

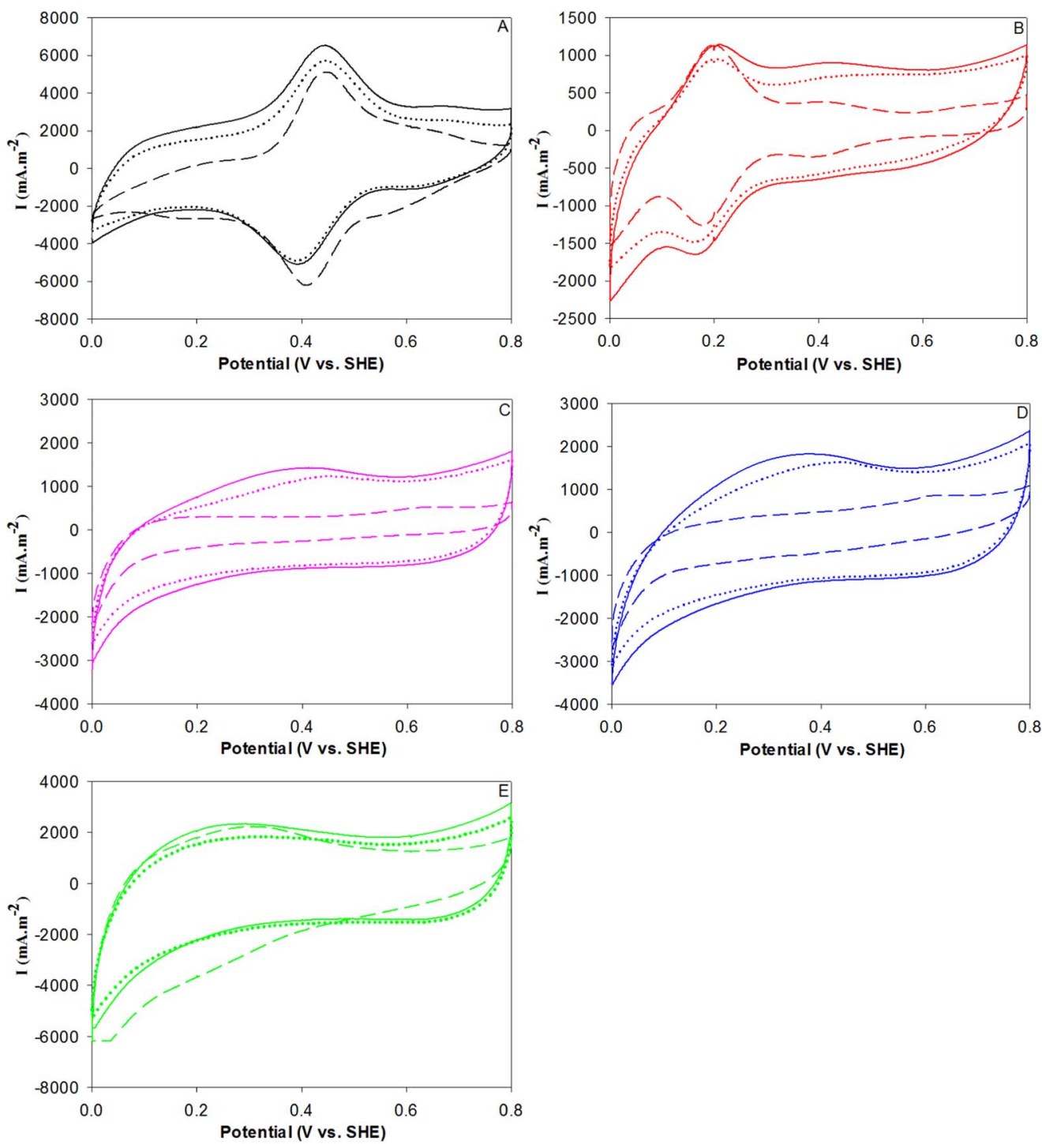

Figure 2. Anodic cyclic voltammograms with immobilized polymers: (A) Os-1; (B) Os-2, (C) polythionine, (D) PMB and (E) PMG, in the absence (long-dashedlines) and presence of the biological consortium: day (solid lines) and night (dotted lines).

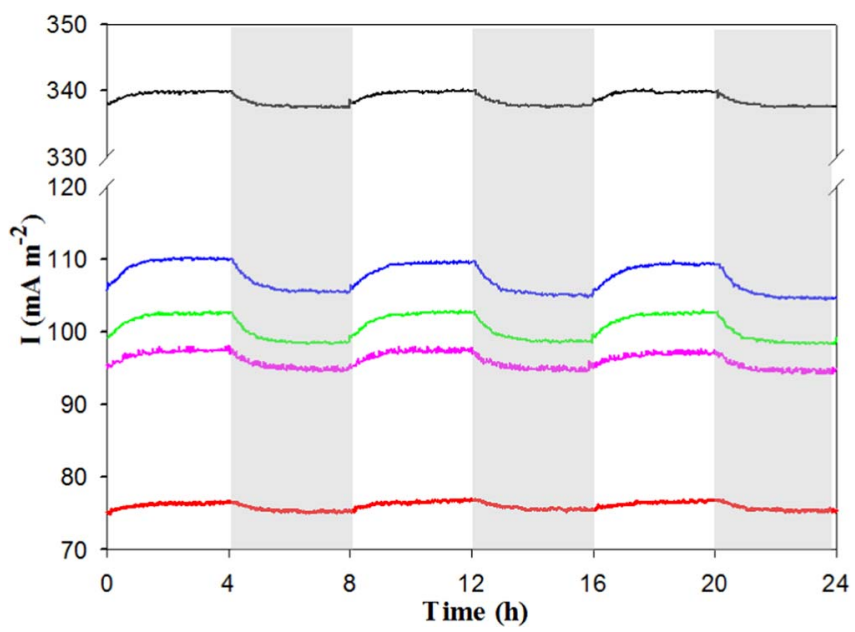

Figure 3. Chronoamperometric anodic current profile of first modified electrochemical cells: Os-1 (black), Os-2 (red), polythionine (pink), PMB (blue) and PMG (green) in presence of the biological consortium.

\section{Acknowledgments}

This work was funded by Australian Research Council grant DE130101168. Libertus Darus is funded by the Directorate General of Higher Education (DGHE) of Indonesia and Australian Research Council (ARC) DP120104415. Pablo Ledezma is funded by ARC DP120104415. Isioma Osadebe is funded by the National University of Ireland Galway. Dónal Leech is funded by the European Commission ("Bioenergy" FP7-PEOPLE-2013-ITN-607793). Lo Gorton is funded by the Swedish Research Council (project 2014-5908) and The European Commission ("Bioenergy" FP7-PEOPLE-2013ITN-607793). Stefano Freguia is funded by the fellowship ARC DE130101168.

\section{References}

1. A. J. McCormick, P. Bombelli, R. W. Bradley, R. Thorne, T. Wenzel, and C. J. Howe, Energy Environmental Science, 8, 1092 (2015).

2. Z. He, J. Kan, F. Mansfeld, L. T. Angenent, and K. H. Nealson, Environmental Science and Technology, 43, 1648 (2009).

3. D. P. B. T. B. Strik, H. V. M. Hamelers, and C. J. N. Buisman, Environmental Science and Technology, 44, 532 (2010).

4. L. Darus, Y. Lu, P. Ledezma, J. Keller, and S. Freguia, Bioresource Technology, 195, 248 (2015). 
5. G. M. Delaney, H. P. Bennetto, J. R. Mason, S. D. Roller, J. L. Stirling, and C. F. Thurston, Journal Chemical Technology Biotechnology, 34, 13 (1984).

6. D. H. Park and J. G. Zeikus, Applied Environmental Microbiology, 66, 1292 (2000).

7. M. Rosenbaum, Z. He, and L. T. Angenent, Current Opinion Biotechnology, 21, 259 (2010).

8. S. Tsujimura, A. Wadano, K. Kano, and T. Ikeda, Enzyme and Microbial Technology, 29, $225(2001)$.

9. K. Nishio, R. Nakamura, X. Lin, T. Konno, K. Ishihara, S. Nakanishi, and K. Hashimoto, ChemPhysChem, 14, 2159 (2013).

10. S. Patil, C. Hägerhäll, and L. Gorton, Bioanalytical Reviews, 4, 159 (2012).

11. I. Osadebe and D. Leech, ChemElectroChem, 1, 1988 (2014).

12. Y. Yuan, H. Shin, C. Kang, and S. Kim, Bioelectrochemistry, 108, 8 (2016).

13. A. A. Karyakin, E. E. Karyakina, W. Schuhmann, and H.-L. Schmidt, Electroanalysis, 11, 553 (1999).

14. J. M. Bauldreay and M. D. Archer, Electrochimica Acta, 28, 1515 (1983).
15. R. Yang, C. Ruan, and J. Deng, Journal of Applied Electrochemistry, 28, 1269 (1998).

16. J. Du, C. Catania, and G. C. Bazan, Chemistry of Materials, 26, 686 (2014).

17. K. Hasan, H. Bekir Yildiz, E. Sperling, P. Ò Conghaile, M. A. Packer, D. Leech, C. Hägerhäll, and L. Gorton, Physical Chemistry Chemical Physics, 16, 24676 (2014).

18. K. Hasan, E. Çevik, E. Sperling, M. A. Packer, D. Leech, and L. Gorton, Advanced Energy Materials, 5, \#1501100 (2015).

19. L. Darus, P. Ledezma, J. Keller, and S. Freguia, Photosynthetic Research, 127, 347 (2015).

20. T. J. Ohara, R. Rajagopalan, and A. Heller, Analytical Chemistry, 65, 3512 (1993).

21. L. Darus, P. Ledezma, J. Keller, and S. Freguia, Environmental Science and Technology, 48, 14000 (2014).

22. A. S. N. Murthy and K. S. Reddy, Electrochimica Acta, 28, 473 (1983).

23. D. MacAodha, M. L. Ferrer, P. Ò Conghaile, P. Kavanagh, and D. Leech, Physical Chemistry Chemical Physics, 14, 14667 (2012).

24. S. Tsujimura, K. Murata, and W. Akatsuka, Journal of the American Chemical Society, 136, 14432 (2014) 